\title{
Is CBD A Viable Option for Menstrual Symptoms?
}

\author{
Jackie P Cavner*, Fort Smith and Brooke Gray \\ Department of Nursing, University of Arkansas, USA
}

*Corresponding author: Jackie Cavner, Carolyn McKelvey Moore School of Nursing, University of Arkansas, Fort Smith, USA.

Received Date: October 24, 2019

Published Date: November 19, 2019

\begin{abstract}
The purpose of this paper is to review relevant studies on the viability of using cannabidiol (CBD) as a treatment option for PMS. Results of this review show improvement of discomforts such as headaches, emotional and mental symptoms, as well as pain. It is concluded that CBD is an alternative treatment option for menstrual symptoms. Limitations were discovered that quality research is deficient. More studies are warranted on using CBD to treat symptoms of PMS and the potential side effects.
\end{abstract}

Keywords: Cannabidiol; CBD; Menstruation; Menstrual pain; Premenstrual syndrome; PMS treatment

Abbreviations: PMS: Premenstrual Syndrome; NSAIDs: Nonsteroidal anti-inflammatory drugs; MI: Myocardial infarction; CBD: Cannabidiol; THC: Tetrahydrocannabinol; ECS: Endocannabinoid system; USDHHS: U.S. Department of Health \& Human Services

\section{Introduction}

Menstruation is the monthly, cyclic occurrence of ovarian and endometrial changes within a woman's body. As the growing follicle matures, the endometrium thickens in preparation for implantation of a fertilized egg. If the egg is not fertilized, the endometrium undergoes a menses where the endometrium sloughs off the uterine wall. This hormonal and physiological process can contribute to subjective and objective symptoms which can include, but are not limited to headaches, mood changes, uterine cramping, as well as abdominal and back pain; otherwise known as Premenstrual Syndrome (PMS). In some women, the pain of menstruation is so pronounced that it affects their daily lives. The term dysmenorrhea is the diagnosis reserved for those women who require pharmacological treatment for the pain [1].

The gold standard for treating such pain and discomfort has been nonsteroidal anti-inflammatory drugs (NSAIDs) for decades. NSAIDs are useful when treating headaches and uterine cramping; however, there is no efficacy on the treatment of the psychological symptoms that often occur. At the same time, NSAIDs do have a black box warning for serious cardiovascular side effects such as myocardial infarction (MI) and stroke. Likewise, serious gastrointestinal problems can occur such as bleeding, ulceration, and perforation [2]. While there are other pharmacological options for treating the plethora of issues accompanied by the menstrual cycle, the purpose of this paper is to review relevant studies on the viability of using cannabidiol (CBD) as a treatment option for PMS.

\section{Discussion}

\section{How it works}

Cannabidiol (CBD) is a substance or compound that derives from the cannabis plant. Unlike delta-9 tetrahydrocannabinol (THC), another compound derived from the same plant, CBD offers potential symptom relief that does not include the psychoactive reaction the THC often comprises. The human body has specific cannabinoid receptors called the $\mathrm{CB} 1$ and $\mathrm{CB} 2$. The $\mathrm{CB} 1$ receptors are located in various regions of the body; however, there is a concentration in the central nervous system and brain. The CB2 receptors are more commonly found in the immune system. The human body produces its own cannabinoids for which these receptors are utilized. It was thought that CBD triggers these receptors after it is taken; however, the body seems to be directed by the CBD to take up more of its own cannabinoids. This entire system is commonly referred to as the endocannabinoid system (ECS). With that said, one can imagine if there are receptors in the brain and immune system then the benefits could be astounding with all that is controlled by the brain: pain perception, coordination, emotions/mood, memories etc. The immune system affects also help to address inflammation and pain [3]. 
There are several forms of CBD products available. The most common forms of CBD use include oils and tinctures, creams and lotions, capsules and pills, edibles, and vaping. Benson [4] The oils and tinctures can be placed in drinks or held under the tongue for sublingual absorption. Pills and capsules are swallowed, but this route takes the longest time to be effective. Creams and lotions are used topically and typically for localized joint pain. Vapors are inhaled and absorbed from the lungs into the bloodstream and this is the fastest route to get relief of symptoms [4].

As with all medical treatments, there are some limitations and possible side effects. There is research available that suggests that the ECS, once stimulated during the ovulatory period of rats, can have an effect on the reproductive tract. However, due to the lack of human studies, it is unclear what effect CBD would have on fertility or the hypothalamic pituitary gonadal axis [5]. Walker 0, et al. [6] also conclude that there is evidence to support that the use of exogenous cannabinoids does effect the ECS and therefore may cause dysregulation of female reproductive process.

\section{Headaches}

There are studies available that have proven cannabinoids can decrease the pain from headaches as well as the frequency of headaches. Rhyne D, et al. [7] states that migraine headache frequency does decrease with the use of medical marijuana, which contains high doses of THC. Cannabinoids are a common treatment modality for headaches as mentioned [8]. This study found that of the patients treating themselves with cannabinoids for pain, $88 \%$ were suffering from migraine headaches. These studies do not differentiate THC and CBD; therefore, more research is needed on CBD only and treating headaches associated with PMS. Piper B, et al. [9] states that $66.7 \%$ of their population that disclosed having migraines had decreased their use of medications after initiating medical marijuana use. In one randomized controlled, double blind study nabilone, a synthetic cannabinoid, was compared to ibuprofen for efficacy. While both the drugs resulted in statistically significant improvement in all primary outcomes, nabilone was superior to ibuprofen [10].

\section{Emotional and mental symptoms}

Premenstrual syndrome symptoms are not limited to physical ailments. Emotional and mental disturbances can bejustas prevalent. This category of symptoms in PMS include mood swings, anxiety and depression, and sleep disturbances including insomnia. [11]. There is evidence suggesting that CBD is a viable anxiolytic. There are subjective data studies comparing CBD to other medications having an equal or positive effect on decreasing anxiety. As well as objective data studies of neuroimaging showing decreased activity in limbic and para-limbic regions during emotional tasks [12]. Shannon et al. [13] confirmed that CBD decreased anxiety scores of $79.2 \%$ of the study population in the first month of use. The scores remained low throughout the study. Dios M, et al. [4 also concluded that marijuana use in young adult females significantly reduces tension and stress. There is some evidence that THC use can induce some anxiety. However, once that evidence was critically appraised and a meta-analysis was complete on high quality research, there was no statistical significance on THC causing anxiety [15]. With that being said, it is important to differentiate between THC and CBD in future studies. THC seems to possibly have some anxiety provoking risks, while CBD seems to relieve such symptoms.

Cannabis and CBD have been recognized for their ability to "alleviate sleeplessness, stress, inflammation, and lingering anxiety" particularly in the form of bath-bombs, or CBD infused bath salts. Galatio K [16] Studies have shown effectiveness in treating symptoms associated with anxiety and sleep disorders, along with arthritis and headaches, when using a high concentration of CBD products alone and with THC compounds. One study showed an improvement of quality of life symptoms including sleep problems up to $29 \%$ after four months of use. [17].

\section{Menstrual pain}

Using cannabis for treating pain is not a new concept. In fact, the use of cannabis for treating pain dates back 5000 years when Egyptians used it to treat neuralgia, headaches, and toothaches [18]. Focusing on the use of CBD for treatment of menstrual pain may be rising in popularity. CBD's pain relieving and antiinflammatory properties could contribute to treating specific menstrual discomforts such as low back pain and lower abdominal cramps. Cannabis and CBD have both been proven to relieve the same symptoms. According to Teitelbaum J [19], there have been multiple studies showing patients found relief of joint pain, fibromyalgia pain, migraines, and spinal pain. "THC has twenty times the potency of aspirin and twice that of hydrocortisone" [19]. There were no studies found specific to menstrual cramps; however, there are adequate studies to support acute and chronic pain relief.

\section{Conclusion}

CBD compounds have been studied and proven to treat and relieve symptoms such as chronic pain, inflammation, anxiety and depression, and insomnia, to name a few [20]. Since many of these symptoms often present themselves to women who suffer from PMS, CBD may be an appropriate, natural alternative treatment when it comes to managing these occurrences. It is clear that more research is needed on the use of CBD to treat PMS specific symptoms. There is a lack of studies on CBD only treatments that do not include THC. There is also a lack of knowledge on possible side effects of CBD. However, the findings of this review conclude that $\mathrm{CBD}$ is a viable option for managing menstrual symptoms.

\section{Acknowledgements}

James R Cavner III Pharm D, Pharmacy Manager, Sam's Club Pharmacy.

Carolyn Fillippelli, University of Arkansas - Fort Smith Library Liaison for the College of Health Sciences.

\section{Conflict of Interest}

Ekaterina Smith, Associate Editor for the Online Journal of Complementary and Alternative Medicine is the authors' coworker at the University of Arkansas - Fort Smith. She has not seen this mini review. 


\section{References}

1. Decherney A, Nathan L, Laufer N, Roman A (2013) Current diagnosis and treatment: Obstetrics and Gynecology (11 ${ }^{\text {th }}$ edn). McGraw Hill Companies, INC. USA, pp. 611-614.

2. Sam s Club Pharmacy (2019) Facts and comparisons eAnswers: Naproxen oral. Obtained from pharmacy manager James Cavner PharmD.

3. Johnson J (2018) Everything you need to know about CBD oil.

4. Benson D (2019) Should CBD be part of my patients' treatment? Alternative Therapies in Health and Medicine 25(1): 8-11.

5. Cooper Z, Craft R (2018) Sex-dependent effects of cannabis and cannabinoids: A translational perspective. Neuropsychopharmacology 43(1): 34-51.

6. Walker O, Holloway A, Raha S (2019) The role of the endocannabinoid system in female reproductive tissues. J Ovarian Res 12(3): 1-10.

7. Rhyne D, Anderson S Gedde M, Borgelt L (2016) Effects of medical marijuana on migraine headache frequency in the adult population. Pharmacotherapy 36(5): 505-510.

8. Baron E, Lucas P, Eades J, Hogue O (2018) Patterns of medicinal cannabis use, strain analysis, and substitution effect among patients with migraine, headache, arthritis, and chronic pain in a medicinal cannabis cohort. J Headache Pain 19(1): 1-37.

9. Piper B, Dekeuster R, Beals M, Cobb C, Burchman C, et al. (2017) Substitution of medical cannabis for pharmaceutical agents for pain, anxiety, and sleep. J Psychopharmacol 31(5): 569-575.

10. Pini L, Guerzoni S, Cainazzo M (2012) Nabilone for the treatment of medication overuse headache: results of a preliminary double-blind, active-controlled, randomized trial. J Headache Pain 13(8): 677-684.
11. US Department of Health \& Human Services (USDHHS) (2018) Premenstrual syndrome.

12. Turna J, Patterson B, Ameringen M (2017) Is cannabis treatment for anxiety, mood, and related disorders ready for primetime? Depress Anxiety 34(11): 1006-1017.

13. Shannon S, Lewis N, Lee H, Hughes S (2019) Cannabidiol in anxiety and sleep: A large case series. Perm J 23: 18-41.

14. Dios M, Hagerty C, Herman D, Hayaki J, Anderson B, et al. (2010) General anxiety disorder symptoms, tension reduction, and marijuana use among young adult females. J Womens Health (Larchmt) 19(9): 16351642.

15. Twomey C (2017) Association of cannabis use with the development of elevated anxiety symptoms in the general population: a meta-analysis. J Epidemiol Community Health 71(8): 811-816.

16. Galatio K (2019) Different CBD methods: Pros and cons. Alternative Therapies in Health \& Medicine 25: 34-35.

17. Wan BA, Diaz P, Blake A, Chan S, Wolt A, et al. (2017) Efficacy of different varieties of medical cannabis in relieving symptoms. Journal of Pain Management 10(4): 375-383.

18. Philippon J (2016) Cannabinoid system: A safer chronic pain management approach. International Student. Journal of Nurse Anesthesia 15(2): 78-86.

19. Teitelbaum J (2019) A hemp oil, CBD, and marijuana primer: Powerful pain, insomnia, and anxiety-relieving tools! Altern Ther Health Med 25(S2): 21-23.

20. Project CBD (2019) What is CBD? 